\title{
Necessidades hídricas da cana-de-açúcar cultivada em clima tropical
}

\section{Water requirements of sugar cane grown in tropical environment}

\author{
Vicente de Paulo Rodrigues da Silva ${ }^{1 *}$; Cicera Josefa Rozangela Borges²; \\ Walker Gomes de Albuquerque ${ }^{3}$
}

\section{Resumo}

A estimativa precisa do consumo hídrico da cana-de-açucar é importante para obtenção da máxima produtividade com menos investimentos. O objetivo do trabalho foi determinar as necessidades hídricas da cana-de-açúcar cultivada em clima tropical. O experimento foi realizado no Estado da Paraíba, durante o ciclo produtivo da cultura da cana-de-açucar variedade RB 92579 irrigada por pivô central, no período de outubro de 2009 a setembro de 2011. A evapotranspiração da cultura (ETc) foi obtido pelo método do balanço hídrico $(\mathrm{BH})$ e a evapotranspiração de referência (ETo) pelo método de PenmanMonteith, utilizando-se os dados de temperatura do ar, umidade relativa, velocidade do vento e radiação solar coletados na Plataforma de Coleta de Dados próxima à area experimental. A umidade do solo foi monitorada utilizando-se sondas TDR (Frequency Domain Reflectometry), modelo PR2/6, Delta-T. Os resultados permitiram constatar que os valores dos coeficientes de cultivo $(\mathrm{Kc})$ propostos pela FAO para a cultura da cana-de-açúcar não são apropriados para regiões tropicais. O consumo hídrico da cultura variou entre $2,6 \mathrm{~mm} \mathrm{dia}^{-1}$, na fase inicial, a $6,38 \mathrm{~mm} \mathrm{dia}^{-1}$, na fase intermediária, com média de $4,3 \mathrm{~mm} \mathrm{dia}^{-1}$. Similarmente, o coeficiente da cultura variou entre 0,56 e 1,43, com média igual a 0,99 , respectivamente, nos mesmos estádios fenológicos da cultura.

Palavras-chave: Balanço hídrico, Saccharum spp, umidade do solo, irrigação, equação de PenmanMonteith

\begin{abstract}
An accurate estimation of water consumption of cane sugar crop is important for maximum productivity with less investment. The objective of this study was to determine the water requirements of sugar cane grown in tropical environments. The field experiment was carried out in Paraiba state, during the productive cycle of sugar cane crop variety RB 92579 irrigated by central pivot irrigation (sprinkler) from October 2009 to September 2011. The crop evapotranspiration was obtained based on soil water balance and reference evapotranspiration by Penman-Monteith method (FAO/56), using the data of air temperature, relative humidity, wind speed and solar radiation from Data Collection Platform, next to the experimental site. Soil water moisture was monitored by TDR probes (Frequency Domain Reflectometry), Model PR2/6, Delta-T. The results showed that the crop coefficients values proposed by the FAO for sugar cane are not suitable for tropical regions. The water consumption of sugar cane ranged from $2.6 \mathrm{~mm} \mathrm{day}^{-1}$, at initial crop growth stage, to $6.38 \mathrm{~mm} \mathrm{day}^{-1}$, with an average of $4.3 \mathrm{~mm}$ day $^{-1}$ for all cycle. Similarly, the crop coefficient ranged from 0.56 to 1.43 , with an average of 0.99 for the same development crop growth stages.
\end{abstract}

Key words: Water balance, Saccharum officinarum L., soil water content, Penman-Monteith approach

\footnotetext{
${ }^{1}$ Prof. Dr. da Universidade Federal de Campina Grande, UFGD, Unidade Acadêmica Ciências Atmosféricas, Campina Grande, PB. E-mail: vicente@dca.ufcg.edu.br

${ }^{2}$ Prof Dr $^{\mathrm{a}}$ da Faculdade Maurício de Nassau, Campina Grande, PB. E-mail: cicera@dca.ufcg.edu.br

${ }^{3}$ Prof. Dr. da UFGD, Campus de Pombal, Pombal, PB. E-mail: walkergomes@yahoo.com.br

* Autor para correspondência
} 


\section{Introdução}

No Nordeste brasileiro as regiões tradicionalmente canavieiras, como as regiões dos Tabuleiros Costeiros e Zona da Mata, não dispõem mais de área para expansão, tornando-se imprescindível o manejo adequado dessa cultura visando o aumento de sua produtividade. Isso implica em conhecer os padrões de crescimento de cada variedade da cultura, fazendo com que as fases de máximo desenvolvimento coincidam com os períodos de maior disponibilidade hídrica e radiação solar, permitindo que ela expresse todo seu potencial genético (KEATING et al., 1999; STONE; SORENSEN; JAMIESON, 1999). A cana-de-açúcar é a principal matéria-prima para a fabricação do açúcar e álcool (etanol). Essa planta é cultivada, principalmente, em clima tropical onde se alternam as estações secas e úmidas. Nesse sentido, a estimativa precisa do consumo hídrico dessa cultura se torna uma tarefa cada vez mais importante no sentido de se obter a máxima produtividade com menos investimentos no seu manejo.

A determinação da evapotranspiração (ET) contribui para a utilização mais racional da água em uma determinada cultura em que as fases críticas de desenvolvimento vegetativo e reprodutivo são limitadas pelo fator hídrico (CUNHA; BERGAMASCHI; BERLATO, 1996). $\mathrm{O}$ planejamento de irrigação baseado em valores empíricos do coeficiente de cultivo $\left(\mathrm{K}_{\mathrm{c}}\right)$ refletem nos custos de produção, na qualidade do produto e no rendimento. Por outro lado, o excesso d'água aplicado às culturas pode provocar salinização do solo e contaminação do lençol freático. Neste sentido, muitos estudos têm sido conduzidos para se obter Kc mais apropriados de acordo com as condições locais, utilizando diferentes técnicas, tais como lisímetro (LÓPEZ-URREA; OLALLA; LÓPEZ-FUSTER, 2009), método do balanço de energia (BORGES et al., 2008) e o método do balanço hídrico (CAMPOS et al., 2007).

O volume de água de que a cultura da canade-açúcar necessita para atingir bons níveis de produtividade varia de 1.500 e $2.500 \mathrm{~mm}$ (OMETTO, 1980). Além disso, a resposta de produtividade da cana-de-açúcar irrigada depende de um conjunto de fatores, destacando-se a quantidade de água, de fertilizantes, manejo de irrigação, da cultivar, idade de corte, do tipo de solo e do clima (THORBURN et al., 2003; RAMESH; MAHADEVASWAMY, 2000; SMIT; SINGELS, 2006).

$\mathrm{O}$ crescimento no setor sucroalcooleiro gera o questionamento sobre os impactos no ambiente, a necessidade do aumento da produtividade e a maior eficiência no uso da água pela cultura. Nesse aspecto, o método que possibilita o estudo mais detalhado das condições hídricas em que a cultura se desenvolveu é o que quantifica os processos da equação do balanço da água no solo (ANTONINO et al., 2000; CINTRA; LIBARDI; SAAD, 2000; TIMM et al., 2002; AZEVEDO; SILVA; SILVA, 2003; CRUZ et al., 2005). Neste sentido, o objetivo deste trabalho é determinar as necessidades hídricas da cana-de-açúcar cultivada em ambientes tropicais, com vistas à utilização apropriada dos recursos hídricos da região e o aumento da produtividade da cultura.

\section{Material e Métodos}

O experimento foi realizado na Miriri Alimentos e Bioenergia S/A, durante o ciclo produtivo da cultura da cana-de-açucar (Saccharum officinarum L.), no período de 10 de outubro de 2009 a 10 setembro de 2011. A variedade de cana-de-açúcar estudada foi a RB 92 579, muito difundida na região Nordeste do Brasil. A Miriri Alimentos e Bioenergia S/A está situada geograficamente na latitude $6^{\circ} 56^{\prime}$, longitude $35^{\circ} 07^{\prime}$, com altitude de $103 \mathrm{~m}$ e temperatura média de $28^{\circ} \mathrm{C}$. A precipitação média anual na região é superior a $1500 \mathrm{~mm}$, com seis meses secos; o clima é quente e úmido, com chuvas de outono a inverno, com seca atenuada (SILVA, 2004).

$\mathrm{O}$ balanço hídrico $(\mathrm{BH})$ foi realizado numa parcela irrigada com base em 100\% da evapotranspiração referência (ETo) cujos dados para 
a sua determinação foram obtidos na Plataforma de Coleta de Dados (PCD) do Instituto Nacional de Pesquisas Espaciais (INPE), localizada na Destilaria Miriri, no Estado da Paraíba, cerca de 300 metros da área experimental.

A evapotranspiração da cana-de-açúcar $\left(\mathrm{ET}_{\mathrm{c}}\right)$ foi determinada pelo método do $\mathrm{BH}$, expresso pela equação:

$$
\mathrm{ETc}=\mathrm{P}+\mathrm{I}+\mathrm{A}-\mathrm{D} \pm \Delta \mathrm{W} \pm \mathrm{RO}
$$

em que $\mathrm{P}$ é a precipitação pluviométrica; I a lâmina de irrigação; A a ascensão capilar; D a drenagem profunda; $\Delta \mathrm{W}$ a variação da lâmina de água disponível no solo e RO o escoamento superficial. Todos os componentes da Eq. (1) são expressos em $\mathrm{mm} \mathrm{d}^{-1}$. O escoamento superficial foi considerado nulo, visto que a topografia do terreno era plana, $\mathrm{P}$ foi monitorada com um pluviômetro instalado na área experimental e $\Delta \mathrm{W}$ determinado com base no perfil de umidade do solo.

O volume de controle considerado para elaboração do balanço hídrico correspondeu a camada de solo compreendida entre a superfície e a profundidade efetiva do sistema radicular. A partir de três trincheiras abertas no interior da parcela, foi determinada a profundidade média do sistema radicular da cultura que foi de 0,6 metros. Como o lençol freático na área de estudo é superior a 1 $\mathrm{m}$ de profundidade, o termo ascessão capilar foi considerado nulo e, também, como não foram instalados tensiômetros para monitorar a tensão de água no solo, a percolação foi obtida com base na equação (ALLEN et al., 1998):

$$
\mathrm{DP}_{\mathrm{i}}=\left(\mathrm{P}_{\mathrm{i}}-\mathrm{RO}_{\mathrm{i}}\right)+\mathrm{I}_{\mathrm{i}}-\mathrm{Dr}_{\mathrm{i}-1}
$$

em que $\mathrm{P}_{i}$ é a precipitação, $\mathrm{RO}_{\mathrm{i}}$ escoamento superficial, $\mathrm{I}_{\mathrm{i}}$ a irrigação do dia i e $\mathrm{Dr}_{\mathrm{i}-1}$ é a depleção da zona radicular no fim dia anterior, i-1 (mm). A depleção inicial foi derivada a partir da quantidade de água no solo por: (ALLEN et al., 1998).

$$
\operatorname{Dr}_{\mathrm{i}-1}=1000\left(\theta_{\mathrm{CC}}-\theta_{\mathrm{i}-1}\right) \mathrm{Z}_{\mathrm{r}}
$$

em que $\theta_{\mathrm{CC}}=$ umidade do solo à capacidade de campo em $\left(\mathrm{m}^{3} / \mathrm{m}^{3}\right), \theta_{\mathrm{i}-1}=$ umidade de solo médio na zona radicular em $\left(\mathrm{m}^{3} / \mathrm{m}^{3}\right), \mathrm{Z}_{\mathrm{r}}=$ Profundidade radicular $(\mathrm{m})$. O armazenamento de água no solo (W), em mm, durante um dado intervalo de tempo foi calculado usando-se a seguinte equação

$$
\mathrm{W}_{\mathrm{t}}=\int_{0}^{\mathrm{L}} \theta(\mathrm{z}) \mathrm{dz}=\bar{\theta} \mathrm{L}
$$

em que $\bar{\theta}$ é a umidade do solo média até a profundidade considerada $\left(\mathrm{cm}^{3} \mathrm{~cm}^{-3}\right)$ e L a espessura da camada de solo $(\mathrm{cm})$. Durante o intervalo de tempo considerado, a variação do armazenamento de água no solo $(\Delta \mathrm{W})$, expressa em $\mathrm{cm}$, foi obtida como

$$
\Delta \mathrm{W}=\mathrm{W}_{\mathrm{t}}-\mathrm{W}_{\mathrm{t}-1}
$$

em que $\Delta \mathrm{W}_{\mathrm{t}} \mathrm{e} \Delta \mathrm{W}_{\mathrm{t}-1}$ representam os armazenamentos de água no perfil do solo nos instantes t e t-1, respectivamente.

A umidade do solo foi monitorada utilizando-se sondas Frequency Domain Reflectometry (TDR), modelo PR2/6, Delta-T (Devices Ltd., Burwell, Cambridge, UK), com três tubos de acesso, instalados a $0,20 \mathrm{~m}$ de cada ponto de observação, nas profundidades de 0,$10 ; 0,20 ; 0,30 ; 0,40 ; 0,60$; 0,$70 ; 0,80 ; 0,90$ e $1,0 \mathrm{~m}$. As medições da umidade do solo foram realizadas a cada 2-3 dias durante o ciclo da cultura.

As análises das características físicas e químicas do solo da área experimental foram realizadas com base em amostras indeformadas, coletadas a partir de trincheiras abertas no interior das parcelas experimentais nas camadas de solo: 0,00-0,20; $0,20-0,40 ; 0,40-0,60 ; 0,60-0,80 ; 0,80-1,00 \mathrm{~m}$, com três repetições. As amostras foram utilizadas para determinação da densidade global, textura e a curva de retenção de água no solo. A condutividade hidráulica do solo da área experimental foi determinada com base em amostras coletadas na camada de solo com a maior concentração de raízes. $\mathrm{A} \mathrm{ET}_{\mathrm{o}}$ foi obtida como descrito em Allen et al. (1998), na forma comumente designada como a equação FAO-PM. 
A ETo foi calculada pelo método de PenmanMonteith (FAO/56), considerando-se a resistência estomática de $70 \mathrm{sm}^{-1}$, albedo de $23 \%$ e a altura da cultura hipotética fixada em 0,12m (ALLEN et al., 1998):

$$
\mathrm{ET}_{\mathrm{o}}=\frac{0,408 \Delta\left(\mathrm{R}_{\mathrm{n}}-\mathrm{G}\right)+\gamma\left(\frac{900 \mathrm{U}_{2}}{\mathrm{~T}+273}\right)\left(\mathrm{e}_{\mathrm{s}}-\mathrm{e}_{\mathrm{a}}\right)}{\Delta+\gamma\left(1+0,34 \mathrm{U}_{2}\right)}
$$

em que $\mathrm{ET}_{\mathrm{o}}$ é a evapotranspiração de referência ( $m m$ dia $\left.^{-1}\right), \mathrm{R}_{\mathrm{n}}$ (saldo de radiação) e $\mathrm{G}$ (densidade do fluxo de calor no solo) são expressos em $\mathrm{MJ} \mathrm{m}^{-2} \mathrm{dia}^{-1}$, $\Delta$ é a declinação da curva de saturação do vapor da água $\left(\mathrm{kPa}^{\circ} \mathrm{C}^{-1}\right)$ e $\mathrm{U}_{2}$ é a velocidade do vento (média diária) a $2 \mathrm{~m}$ acima da superfície do solo $\left(\mathrm{m} \mathrm{s}^{-1}\right)$, T é a temperatura do $\operatorname{ar}\left({ }^{\circ} \mathrm{C}\right), \mathrm{e}_{\mathrm{s}}$ é a pressão de saturação do vapor de água $(\mathrm{kPa}), \mathrm{e}_{\mathrm{a}}$ é a pressão real do vapor $(\mathrm{kPa})$ e $\gamma$ é o fator psicrométrico $\left(\mathrm{MJ} \mathrm{kg}^{-1}\right)$.

\section{Resultados e Discussão}

Os componentes do $\mathrm{BH}$ e os Kcs durante os quatro estádios fenológicos do ciclo da cultura da cana-de-açúcar, bem como os períodos de cada estádio fenológico da cultura, são exibidos na Tabela 1. A alta freqüência e a quantidade de irrigação, que durante todo o ciclo da cultura foi de 1.342,2 $\mathrm{mm}$, provocou drenagem elevada $(301,1 \mathrm{~mm})$, que correspondeu a $22,4 \%$ da irrigação total. Por outro lado, a precipitação total do período representou pouco mais de $1 / 3$ da média climatológica da região $(\approx 1500 \mathrm{~mm})$. Esses resultados sugerem que o uso inapropriado do Kc, que produzem altas lâminas de irrigação, associado com a alta freqüência de irrigação, provoca perda excessiva de água para o lençol freático; contribuindo, dessa forma, para a salinização do solo e aumento dos custos de produção; além disso, podem surgir incidências de fungos no solo, acarretando morte de plantas e baixa na produtividade (THORBURN et al., 2003). Adicionalmente, o excesso de água, aliado a um período prolongado de saturação e pouca aeração do solo, pode favorecer o desenvolvimento de alguns patógenos, lixiviação de nutrientes e redução do desenvolvimento radicular, especialmente das raízes adventícias. Os valores médios e acumulados da ET com base no BH são, respectivamente, 4,30 $\mathrm{mm} \mathrm{dia}^{-1}$ e $1.686,75 \mathrm{~mm}$. Os valores da ET média e acumulada são sempre maiores na fase média e menor nas fases inicial e final. Como consequência, o Kc apresenta comportamento similar, com média para todo o ciclo de 0,99 .

No manejo da irrigação usualmente são utilizados para qualquer região os Kc propostos no Boletim 56 da FAO sem qualquer ajuste às condições climáticas locais. Entretanto, esse procedimento muitas vezes é equivocado porque eles são válidos apenas para climas sub úmidos, caracterizados por umidade relativa média mínima diária de $\approx 45 \%$ e moderada velocidade média do vento de $\approx 2 \mathrm{~m} \mathrm{~s}^{-1}$ (ALLEN et al., 1998). Efetivamente, os dados do presente estudo indicam que os valores do Kc baseado no $\mathrm{BH}$ maiores do que aqueles propostos no boletim $\mathrm{FAO} / 56$ praticamente em todas as fases fenológicas da cultura da cana-de-açúcar. Este resultado é consistente com aqueles registrados por Allen et al. (1998), em que o Kc é maior em condições climáticas mais áridas e com altos valores de velocidade do vento do que em climas mais úmidos e com baixa velocidade do vento.

A média semanal da ETc btida com base no $\mathrm{BH}$, e os valores da precipitação + irrigação na parcela experimental são apresentadas na Figura 1. A ETc foi determinada em intervalos de 3-7 dias de forma que a precipitação e/ou irrigação fosse contemplada no cálculo da ET nesse período. Procedimento semelhante foi utilizado por Azevedo, Silva e Silva (2003) quando determinaram a ET da mangueira na região do sub médio Rio São Francisco com base no método do BH. Os menores valores da ET ocorreram no período em que não houve irrigação e máximos nos períodos de maior freqüência e intensidade de irrigação e precipitação. Dessa forma, muito embora a ET da cana-de-açúcar seja fortemente influenciada pelo total de água aplicado à cultura, ela também depende da demanda 
atmosférica e do desenvolvimento da área foliar. A ETc não necessitou de nenhum ajuste, haja vista que a cultura não passou por estresse hídrico em nenhum momento do seu ciclo de produção. Isso era esperado, em face das altas lâminas e freqüências das irrigações.

Tabela 1. Valores médios da evapotranspiração [ET (médio), $\mathrm{mm} \mathrm{dia}^{-1}$ ], acumulados [ET (acumulada), mm], coeficientes de cultivo (Kc), precipitação (Prec, $\mathrm{mm}$ ), irrigação (Irrig, $\mathrm{mm}$ ) e drenagem (Dren, $\mathrm{mm}$ ) durante as fases do ciclo da cultura da cana-de-açúcar.

\begin{tabular}{lccccrrr}
\hline Estádios & \multicolumn{1}{c}{ Data $^{*}$} & ET (médio) & ET(acumulada) & Kc & Prec. & \multicolumn{1}{l}{ Irrig. } & Dren. \\
\hline Inicial & $(10 / 10-08 / 11) / 2009$ & 2,60 & 77,92 & 0,56 & 0,0 & 30,0 & 13,8 \\
Desenvolvimento & $(09 / 11-28 / 12) / 2009$ & 3,33 & 166,36 & 0,65 & 12,0 & 146,9 & 0,0 \\
Média & $29 / 12 / 209-26 / 06 / 2010$ & 6,38 & 1148,92 & 1,43 & 421,0 & 932,2 & 157,5 \\
Final & $(27 / 06-25 / 08 / 2010)$ & 4,89 & 293,55 & 1,32 & 188,0 & 233,1 & 129,8 \\
Total & - & - & 1686,75 & & 621,0 & 1342,2 & 301,1 \\
Média & - & 4,30 & - & 0,99 & & - & - \\
\hline
\end{tabular}

Fonte: Elaboração dos autores.

As variações das médias semanais da ETc obtidas com base no $\mathrm{BH}$ e os desvios padrão são exibidas na Figura 2. Em face da dispersão dos dados e a influência do frequente umedecimento da superfície do solo através de irrigação sobre a ETc, os valores da ET calculados são exibidos em médias de 7 dias. A curva da ETc com base no BH descreve de forma bem homogênia o ciclo da cultura. Observa- se uma inflexão da curva da ETc em torno do dia após emergência (DAE) 150 devido a redução da demanda evaporativa durante o período de chvas na região. De forma geral, a ETc aumenta rapidamente a partir do início do ciclo da cultura até atingir a fase média, como resultado do aumento do dossel da planta, e em seguida decresce em função da redução da frequência da irrigação e do declínio da demanda evaporativa.

Figura 1. Média semanal da evapotranspiração da cana-de-açúcar obtida com base no balanço hídrico no solo e os valores da precipitação + irrigação na parcela experimental.

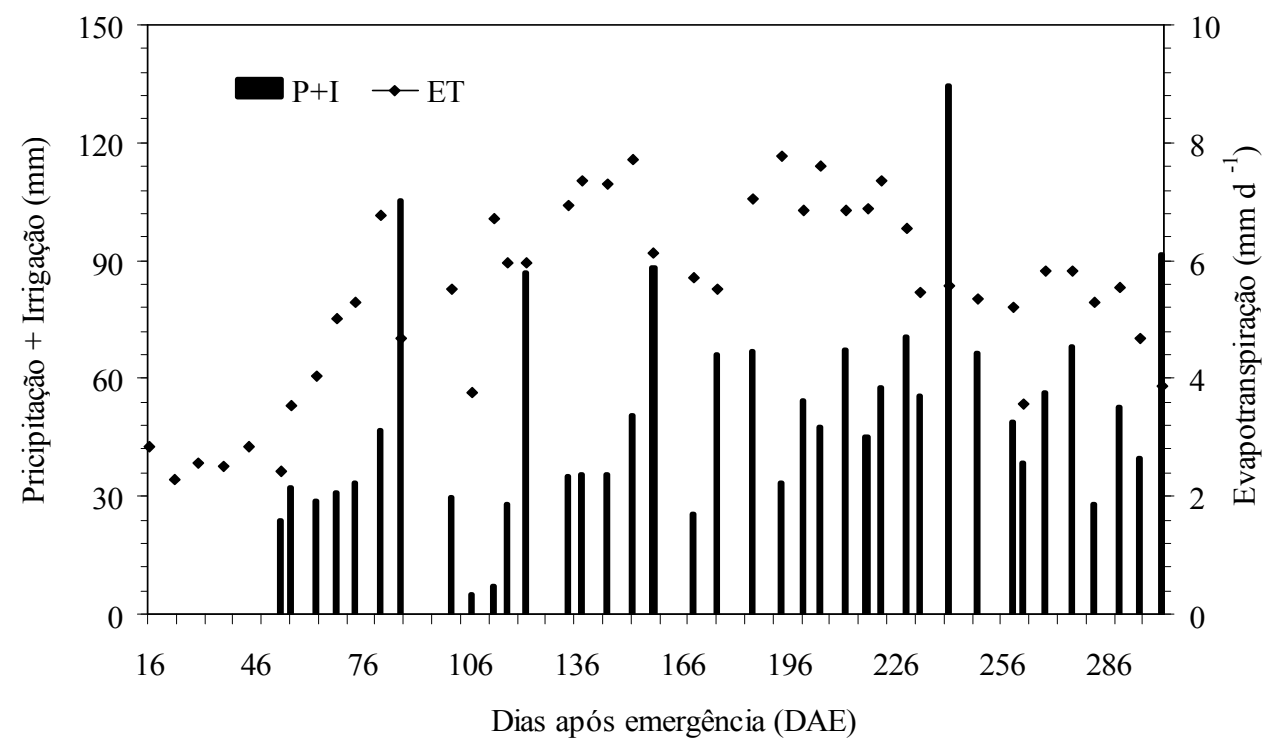

Fonte:Elaboração dos autores. 
Figura 2. Curso sazonal da evapotranspiração da cana-de-açúcar obtido com base no balanço hídrico no solo. Os triângulos significam a média de 7 dias dos valores da evapotranspiração e as barras indicam o desvio padrão.

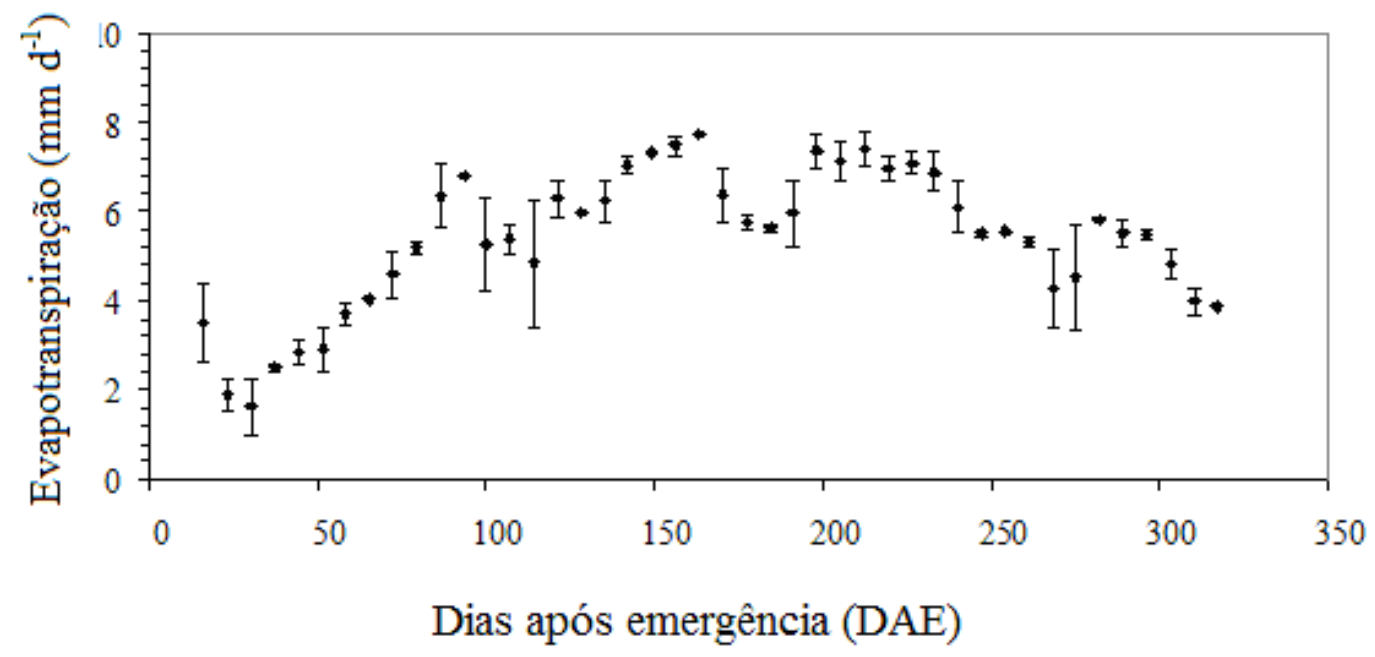

Fonte: Elaboração dos autores.

Figura 3. Curso sazonal da evapotranspiração obtida com base no balanço hídrico no solo (triângulos) e coeficiente de cultivo (linha espessa) da cana-de-açúcar cultivada em condições climáticas tropicais.

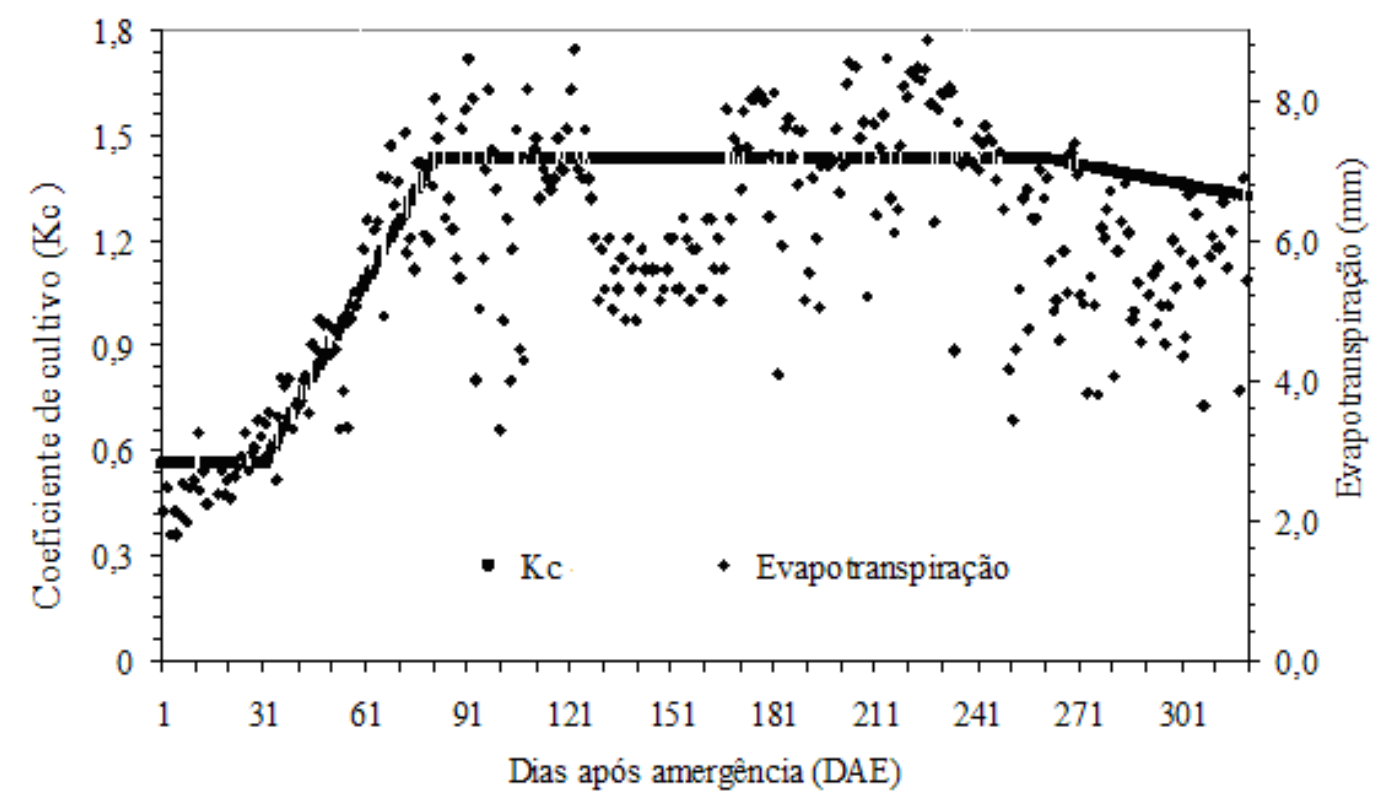

Fonte: Elaboração dos autores. 
O curso sazonal da ET obtida com base no BH e Kc da cana-de-açúcar cultivada nas condições climáticas tropicais é exibido na Figura. 3. Os valores da ETc ao longo do ciclo foram bastante dispersos, porém consistentes com a curva do Kc. Os valores médios do Kc da cana-de-açúcar utilizados para construção da curva do Kc da região nas fases inicial, média e final foram consideravelmente diferentes daqueles propostos pela FAO para a cultura da cana-de-açúcar cultivada em climas sub úmidos. Entretanto, os valores dos Kcs apresentados neste estudo são consistentes com aqueles obtidos por Inman-Bamber e Mcglinchey (2003) quando determinaram o Kc e a eficiência do uso de água da cana-de-açúcar cultivada na Austrália e Suíça usando o método do balanço de energia baseado na razão de Bowen na estimativa da ETc. Esses autores encontraram o Kc da cana-de-açúcar variando entre 0,5 e 1,5, sendo o Kc médio de 1,23; enquanto a ET e o desvio padrão foram de 5,48 $\pm 0,13$. Os valores de Kc e ET da cana-de-açúcar encontrados para esses países de Europa são levemente inferiores daqueles obtidos nesta pesquisa fundamentalmente em face da diferença entre os climas das duas regiões.

\section{Conclusões}

Os valores do coeficiente de cultivo propostos pela FAO para a cultura da cana-de-açúcar não são apropriados para regiões tropicais. Os valores da ET média e acumulada são sempre maiores na fase média e menor nas fases inicial e final da cultura. Como consequência, o Kc apresenta comportamento similar, com média para todo o ciclo de 0,99. A ET da cana-de-açúcar é fortemente influenciada pelo total de água aplicado à cultura, bem como pela demanda atmosférica e desenvolvimento da área foliar da cultura.

\section{Referências}

ALLEN, R. G.; PEREIRA, L. S.; RAES, D.; SMITH, M. Crop evapotranspiration: guidelines for computing crop water requirements. Rome: FAO Irrigation and Drainage Paper 56, 1998. 300 p.

ANTONINO, A. C. D.; SAMPAIO, E. V. S. B.; DALLOLIO, A.; SALCEDO, I. H. Balanço hídrico em solo com cultivos de subsistência no semi-árido do Nordeste do Brasil. Revista Brasileira de Engenharia Agricola e Ambiental,Campina Grande, v. 4, n. 1, p. 2934, 2000.

AZEVEDO, P. V.; SILVA, B. B.; SILVA, V. P. R. Water requirements of irrigated mango orchards in Northeast Brazil. Agricultural Water Management, Amsterdam, v. 58, n. 1, p. 241-254, 2003.

BORGES, C. J. R.; SILVA, V. P. R.; CAMPOS, J. H. B. C.; AZEVEDO, P. V.; MOURA, M. S. B.; SOARES, J. M.; SILVA, B. B. Influência do calor armazenado no sistema solo-planta no balanço de energia em pomar de mangueiras. Revista Brasileira de Engenharia Agrícola e Ambiental, Campina Grande, v. 12, n. 1, p. 393-399, 2008.

CAMPOS, J. H. B. C.; SILVA, V. P. R.; AZEVEDO, P. V.; BORGES, C. J. R.; SOARES, J. M.; MOURA, M. S. B.; SILVA, B. B. Evapotranspiração e produtividade da mangueira sob diferentes tratamentos de irrigação. Revista Brasileira de Engenharia Agrícola e Ambiental, Campina Grande, v. 12, n. 1, p. 150-156, 2007.

CINTRA, F. L. D.; LIBARDI, P. L.; SAAD, A. M. Balanço hídrico no solo para porta-enxertos de citros em ecossistema de Tabuleiro Costeiro. Revista Brasileira de Engenharia Agrícola e Ambiental, Campina Grande, v. 4, n. 1, p. 23-28, 2000.

CRUZ, A. C. R.; LIBARDI, P. L.; CARVALHO, L. A.; ROCHA, G. C. Balanço de água no volume de solo explorado pelo sistema radicular de uma planta de citros. Revista Brasileira de Ciência de Solo, Viçosa, MG, v. 29, n. 1, p. 1-10, 2005.

CUNHA, G. R.; BERGAMASCHI, H.; BERLATO, M. A. Balanço de energia em cultura de milho. Revista Brasileira Agrometeorologia, Santa Maria, v. 4, n. 1, p. 1-14, 1996.

INMAN-BAMBER, N. G.; MCGLINCHEY, M. G. Crop coefficients and water-use estimates for sugarcane based on long-term Bowen ratio energy balance measurements. Field Crops Research, Amsterdam, v. 83, n. 1, p. 125138, 2003. 
KEATING, B. A.; ROBERTSON, M. J.; MUCHOW, R. C.; HUTH, N. I. Modelling sugarcane production systems I: development and performace of the sugarcane module. Field Crops Research, Amsterdam, v. 48, n. 1, p. 27-36, 1999.

LÓPEZ-URREA, R.; OLALLA, F. M. S.; LÓPEZFUSTER, A. M. Single and dual crop coefficients and water requirements for onion (Allium cepa L.) under semiarid conditions. Agricultural Water Management, Amsterdam, v. 96, n. 1, p. 1031-1036, 2009.

OMETTO, J. C. Parâmetros meteorológicos e a cultura da cana-de-açúcar. Piracicaba: ESALQ, 1980. 17 p.

RAMESH, P.; MAHADEVASWAMY, M. Effect of formative phase drought on different classes of shoots, shoot mortality, cane attributes, yield and quality of four sugarcane cultivars. Journal of Agronomy and Crop Science, Malden, v. 185, n. 1, p. 249-258, 2000.

SILVA, V. P. R. On climate variability in Northeast of Brazil. Journal of Arid Environments, Cambridge, v. 58, n. 4, p. 575-596, 2004.
SMIT, M. A.; SINGELS, A. The response of sugarcane canopy development to water stress. Field Crops Research, Amsterdam, v. 98, n. 1, p. 91-97, 2006.

STONE, P. J.; SORENSEN, I. B.; JAMIESON, P. D. Effect of soil temperature on phenology, canopy development, biomass and yield of maize in a cooltemperature climate. Field Crops Research, v. 48, n.1, p. 169-178, 1999.

THORBURN, P. J.; DART, I. K.; BIGGS, I. M.; BAILLIE, C. P.; SMITH, M. A.; KEATING, B. A. The fate of nitrogen applied to sugarcane by trickle irrigation. Irrigation Science, New York, v. 22, n. 1, p. 201-209, 2003.

TIMM, L. C.; OLIVEIRA, J. C. M.; TOMINAGA, T. T.; CÁSSARO, F. A. M.; REICHARDT, K.; BACCHI, O. O. S. Water balance of a sugarcane crop: Quantitative and qualitative aspects of its measurement. Brasileira de Engenharia Agrícola e Ambiental, Campina Grande, v. 6, n. 1, p. 57-62, 2002. 